\title{
Falsche Inhalation immer noch sehr häufig
}

\author{
In den letzten 40 Jahren hat sich die Fehlerquote bei der Inhalation von Asthma- und COPD-Medika- \\ menten kaum verbessert, wie eine Metastudie zeigt. Die Schulung muss dringend verbessert werden.
}

Für einen Review über die Jahre 1975-2014 wurden 144 Arbeiten aus 31 Ländern identifiziert, die sich mit der Inhalationstechnik von Asthma- und COPD-Patienten beschäftigten. In allen wurde die Technik von geschultem Personal direkt beobachtet. Insgesamt beinhalteten die Untersuchungen 54.354 Patienten. Die Anwendungen wurden als korrekt, akzeptabel oder falsch klassifiziert. So konnten die drei häufigsten Fehler identifiziert werden.

Bei den Dosieraerosolen hatten 45\% der Probanden Probleme mit der Koordination, 44\% machten Fehler hinsichtlich der Geschwindigkeit und/oder der Tiefe der Inspiration, 46\% hielten nach der Inhalation nicht den Atem an. Bei den Trockenpulverinhalationen sah es etwas anders aus: $29 \%$ bereiteten das Gerät nicht richtig vor, $46 \%$ atmeten vor der Inhalation nicht vollständig aus, 37\% hielten den Atem nach der Inhalation nicht lang genug an.

Insgesamt lag die Prävalenz der richtigen Anwendung bei 31\%. Bei 41\% war sie akzeptabel und bei $31 \%$ schlecht. Ein Vergleich der Zeiträume 1975-1994 und 1995-2014 ergab keine signifikanten Unterschiede hinsichtlich Fehlerhäufigkeit und Inhalationsqualität.

- Sanchis J, Gich I, Pedersen Set al. Systematic review of errors in inhaler use: has patient technique improved over time? Chest. 2016;150:394-406

\section{KOMMENTAR}

Trotz aller technischen Modifikationen der Devices ist der Anteil von Patienten mit falscher Inhalationstechnik offensichtlich immer noch viel zu hoch. Ein Großteil der Patienten mit Asthma und COPD wird in allgemeinärztlichen Praxen betreut. Hier braucht der Patient mehr als nur das Rezept. Wenn die Technik nicht immer wieder überprüft und in Schulungen aufgefrischt wird, schleichen sich Fehler ein. So kann es so weit kommen, dass unangenehme Nebenwirkungen den Patienten mehr belasten, als er von der richtigen Anwendung der Therapie profitieren könnte.

Prof. Dr. med. H. S. FüeßI

\section{Ringförmige Läsionen auf Stirn und Händen}

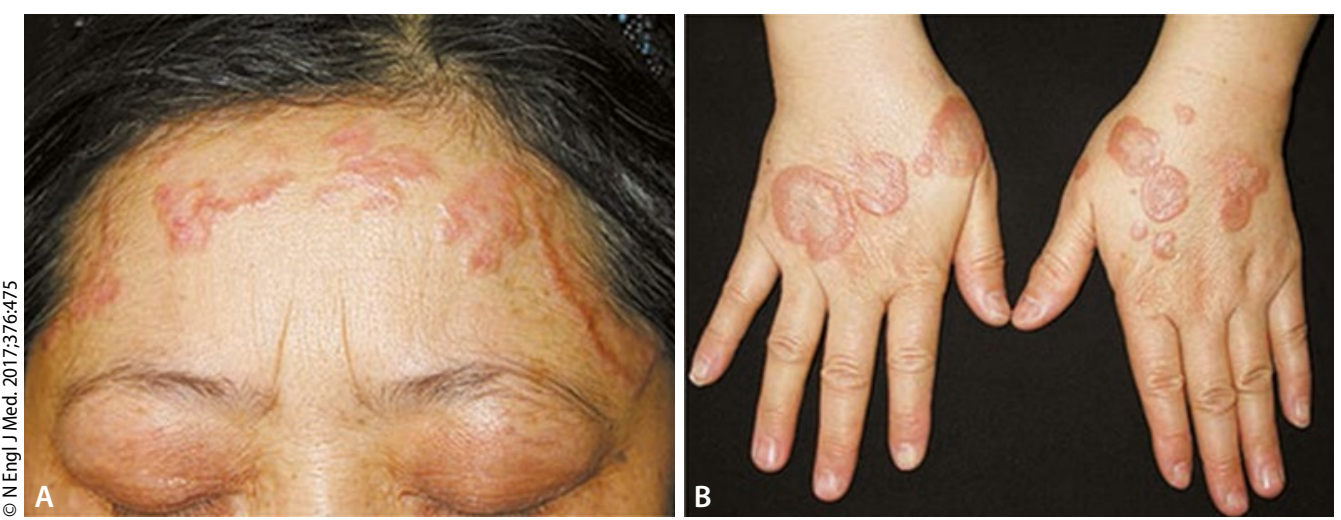

Ringförmige Plaques mit erhabenem Randwall an Stirn und Handrücken.

Die Fragmentation elastischer Fasern und die Phagozytose durch Riesenzellen in den granulomatösen Arealen ließen an ein aktinisches Granulom denken.

Aktinische Granulome sind asymptomatische, granulomatöse Reaktionen im Bereich von sonnenexponierter Haut, die am häufigsten im Gesicht, im Nacken und am behaarten Kopf vorkommen. Sie sind charakterisiert durch ringförmige oder polyzyklisch begrenzte Läsionen, die sich zentrifugal ausbreiten, einen erythematösen Randwall auf-

Eine 50-jährige Frau stellte sich mit Hautveränderungen an der Stirn (Abb. A) und an beiden Handrücken (Abb. B) vor. Die Läsionen waren scharf begrenzt, gerötet und bestanden aus konfluierenden, ringförmigen Plaques mit erhabenem Randwall und leicht atrophischem Zentrum. Sie hatten sich über 18 Monate hinweg langsam ausgebreitet. Eine Hautbiopsie ergab lymphohistiozytäre Infiltrate mit mehrkernigen Riesenzellen und umgebender Elastose ohne Palisadenbildung, Nekrobiose oder Muzindeposition. weisen und zentral unter Hypopigmentierung und Atrophie abheilen. Der Patientin wurde empfohlen, die Sonne zu meiden und Sonnencreme zu benutzen. Unter Therapie mit Halometason-Creme auf den Handrücken und Pimecrolimus-Creme im Stirnbereich bildeten sich die Veränderungen im Verlauf von zwei Monaten teilweise zurück.

Prof. Dr. med. H. S. FüeßI

- Ma DL, Vano-Galvan S (mdonglai@sohu.com). Actinic Granuloma. N Engl J Med. 2017;376:475 